Meta

Journal des traducteurs

Translators' Journal

\title{
Traduction et sociolinguistique
}

\section{Jean-René Ladmiral}

Volume 27, numéro 2, juin 1982

URI : https://id.erudit.org/iderudit/003368ar

DOI : https://doi.org/10.7202/003368ar

Aller au sommaire du numéro

Éditeur(s)

Les Presses de l'Université de Montréal

ISSN

0026-0452 (imprimé)

1492-1421 (numérique)

Découvrir la revue

Citer cet article

Ladmiral, J.-R. (1982). Traduction et sociolinguistique. Meta, 27(2), 196-206.

https://doi.org/10.7202/003368ar

Ce document est protégé par la loi sur le droit d'auteur. L'utilisation des services d'Érudit (y compris la reproduction) est assujettie à sa politique d'utilisation que vous pouvez consulter en ligne.

https://apropos.erudit.org/fr/usagers/politique-dutilisation/ 


\section{APPENDICE}

\section{TRADUCTION ET PSYCHOLOGIE}

JEAN-RENÉ LADMIRAL

All the world's a stage...

SHAKESPEARE

Les rapports entre psychologie et traduction sont, à l'évidence, excessivement nombreux et divers; et l'on comprend qu'à lui seul, le binôme «psychanalyse et traduction" s'avère constituer un champ d'étude déjà fort étendu. Tel n'est pourtant pas l'objet dont nous traitons directement ici $^{1}$ : au lieu $\mathbf{d}^{\prime}$.....analyser la traduction sous les aspects spécifiques d'une psychologie des profondeurs, individuelle(s), c'est la perspective d'une psycholinguistique collective que nous allons adopter. La traduction constitue bien en effet un objet d'étude psychosociologique dans la mesure où, au sein d'un groupe traversé par le clivage des langues, la plupart des individus se trouveront confrontés à l'obligation de traduire, de se traduire ou de se faire traduire, dès lors qu'ils voudront pouvoir communiquer verbalement les uns avec les autres.

Ces diverses opérations de traduction se situent dans le cadre plus général d'une dynamique des groupes bilingues; et c'est cette dernière que prend pour objet la présente étude. Les réflexions que nous y développerons sont issues d'une recherche que nous menons avec l'aide de l'Office franco-allemand pour la jeunesse (O.F.A.J.) depuis quelque trois-quatre ans ${ }^{2}$. Le manque de place nous impose de nous en tenir ici à la présentation de quelques-unes seulement des premières conclusions provisoires auxquelles nous sommes parvenu et il nous est apparu qu'il fallait commencer par exposer les linéaments de cette dynamique des groupes bilingues ${ }^{3}$.

1. Au départ, en effet, ce n'est pas tant à la psychanalyse qu'à la psychologie que semblait devoir être consacré le numéro spécial. Quant au problème des rapports entre psychanalyse et traduction - que nous avons abordé in J.-R. Ladmiral (1979), p. 249 sqq. - nous y reviendrons dans le cadre d'une prochaine étude; mais signalons au passage le brillant article de M. B. de Launay (1981). - N.B. : On trouvera en fin d'article les références bibliographiques complètes des travaux cités.

2. $\grave{A}$ plus court terme, les pages qu'on va lire reprennent aussi en partie la substance de la communication que nous avons présentée au symposion international de Bruxelles sur les langues en contact et en conflit, le 9 juin 1979, sous le titre: "Pouvoir et communication dans les groupes bilingues». Une version écourtée du présent «papier» est publiée parallèlement, en Allemagne, dans le cadre des Actes du symposion de Bruxelles, cf. P. H. Nelde (1980), p. 181-189; ainsi qu'en France, dans la revue de psychosociologie Connexions, $\mathrm{n}^{\circ} 33$ (1981), p. $55-68$

3. De même, l'exposé des problèmes méthodologiques soulevés préalablement par cette recherche ne peut être repris ici de façon détaillée. Nous y avons déjà consacré une étude circonstanciée, à laquelle nous renvoyons le lecteur; et c'est là aussi que nous donnons un historique et une description précise du dispositif de recherche mis en place: J.-R. Ladmiral (1978). Par ailleurs, s'agissant d'un domaine de recherche relativement nouveau, il n'existe guère de littérature concernant directement le sujet dont nous traitons ici et nous n'avons pas voulu multiplier les références à des ouvrages cités faisant plus ou moins fonction d'alliciants: citons seulement P. Scherfer (1977) et E. Marc-Lipiansky (1968); c'est aussi pourquoi nous avons été amené à nous référer surtout à quelques-uns de nos propres travaux. Outre les quelques études traitant de ces questions dans une perspective plutôt psychosociologique que nous avons déjà publiées, nous pouvons annoncer la parution de trois articles prolongeant les pages qu'on va lire, et où nous traitons des problèmes de la traduction dans les groupes: 
Il s'agit en l'occurrence vraiment d'une «situation de laboratoire", bien différente des conjonctures sociolinguistiques qui mettent habituellement deux (ou plusieurs) langues en contact et/ou en conflit. L'objet qui nous occupe ici est l'intervention de la variable linguistique du bilinguisme dans la vie des groupes (au sens des groupes restreints que la psychologie sociale prend pour objet). Concrètement, les groupes étudiés sont des groupes de jeunes Français et de jeunes Allemands, vivant ensemble dans le cadre d'un stage résidentiel, d'une huitaine de jours (éventuellement renouvelable), essentiellement consacré à des activités de loisirs et de formation.

Le dispositif de recherche adopté pour mettre en cuvre ledit programme de recherche franco-allemande dont nous avons la responsabilité, et dont il est question ici, réside donc pour l'essentiel dans l'organisation de stages $^{4}$, réunissant chacun une quinzaine de jeunes Allemands et une quinzaine de jeunes Français, auxquels vient s'adjoindre une équipe d'animation et de recherche (dans le jargon franco-allemand des initiés : «le Team "), elle-même bi-nationale (franco-allemande) et pluri-disciplinaire, c'est-à-dire qu'elle réunit quatre à six chercheurs de disciplines différentes et appartenant aux deux pays. Ainsi dans les équipes que nous avons eu à constituer et/ou auxquelles nous avons participé, on s'est appliqué à faire travailler ensemble des psychosociologues bien sûr mais aussi des ethnologues, ainsi que des philosophes, des sociologues, des psychologues... sans oublier les linguistes, mais sans non plus les privilégier, bien au contraire! Précisons que ces animateurs-chercheurs des deux pays "mis en présence» ne sont pas forcément et pas tous bilingues.

Quant aux participants «de base», ce sont le plus souvent des étudiants (pour des raisons évidentes, de commodité matérielle essentiellement), mais ce sont aussi de jeunes professionnels, plus ou moins engagés dans la vie sociale, voire politique, de leur(s) pays. Matériellement, les frais de stage sont pris en charge par l'Institution commanditaire de la recherche (l'O.F.A.J.).

De tels stages sont dits de «formation-recherche», c'est-à-dire à la fois qu'il s'agit de formation-recherche mais aussi qu'ils fonctionnent simultanément:

- comme élément du dispositif de recherche en ce qui nous concerne, nous "Teamors» ou membres de l'équipe d'animation et de recherche, qui avons aux termes du contrat à fournir un «papier» de recherche.

- et, pour ainsi dire, comme «groupe de contrôle» pour les participants qui, dans la plupart des cas, contribuent à animer eux-mêmes parallèlement des rencontres francoallemandes de base (formation). Il est prévu un suivi de plusieurs stages, permettant de capitaliser les acquis de recherche.

L'hypothèse de départ est que l'introduction de la composante nationale et du bilinguisme redistribue totalement les cartes de la dynamique de groupe classique. C'est, au demeurant, en réponse aux préoccupations de certains praticiens de la rencontre internationale au sein des Mouvements ou Institutions de jeunesse que l'idée de départ nous est venue de mettre sur pied un tel programme de recherche. Certains n'étaient-ils pas allés jusqu'à risquer cette thèse lapidaire : la dynamique de groupe dans un contexte bi-national, ça ne marche pas! (cf. J.-R. Ladmiral, 1978 , p. 7)

En règle générale, il existe un thème, sur lequel est censé travailler le groupe. Mais dans le cas des stages qui nous occupent ici très précisément (ceux dont nous avons eu la

un texte sur le corps et les langues, dans la revue Esprit (sous presse); une Note sur la traduction du rire dans les groupes bilingues, à paraître dans un prochain numéro de la revue francoitalienne Lectures (Université de Bari); et le texte de notre communication sur les problèmes psychosociologiques de la traduction au colloque international de Giessen sur les langues en contact (21-23 février 1980), à paraître dans les Actes édités par J. Caudmont...

4. On notera au demeurant que, d'une façon générale, le dispositif du stage a connu un extraordinaire développement dans les milieux de la formation et de la psychosociologie ces dernières années en France. Pour une présentation, à vrai dire très "orientée » et excessivement critique, de ce phénomène que constitue la multiplication des stages, cf. J. Guigou (1975). C'est aussi, on l'aura deviné, un peu en pensant à cette vogue inflationniste des stages que nous avons choisi notre exergue, par un mauvais calembour «bilingue"... 
responsabilité directe), le thème servait plutôt d' "embrayeur» (shifter) à la dynamique du groupe et d' «analyseur» de ses transversalités et conflits sous-jacents. En effet, il s'est toujours établi un accord pour ne pas aller dans le sens de ce qui était appelé une «académisation" (Akademisierung) et critiqué à ce titre. Au reste, nous avions quant à nous personnellement formulé l'intitulé du thème, tant pour le programme de recherche lui-même que pour chacun des stages mis en place, dans les termes suivants : Identité nationale et dynamique des groupes bilingues. L'idée étant que les problèmes d'identité pourraient servir de point d'ancrage conscient et explicite à la réflexion et aux rationalisations du groupe, à ses projections, etc. mais que l'essentiel se jouerait "objectivement » au niveau de la dynamique réelle dudit groupe, qui est le mode effectif de mise en jeu des identités nationales et linguistiques en présence (en lice?).

C'est ainsi que nous étions en situation de traiter le thème - ou, plutôt, $d u$ thème de toute façon, objectivement, même si par exemple le groupe refusait toute «académisation» et tout discours didactique, et poussait même la contre-dépendance jusqu'à rejeter complètement le thème en tant que tâche explicite (ce qui constituait une façon drastique de «traiter la commande»...). De fait, on se trouvait très souvent confronté à un double paradoxe. Certains (surtout parmi les Français) refusaient donc l'intitulé explicitement didactique du thème et tendaient à centrer le groupe sur ses clivages propres, ce qui était à l'évidence la meilleure façon d'aborder le contenu réel et concret dudit thème. Alors que d'autres (plus souvent parmi les Allemands) finissaient par exploser en récriminations du type: «Assez de dynamique de groupe!» et réclamaient: «Qu'on retourne au thème!», ce qui participait bien évidemment de la même contradiction paradoxale, en l'occurrence plus naïve et «subie» que dans le premier cas de figure.

Quant au mode d'animation adopté (et là nous disons bien : «adopté» plutôt que «choisi »), nous le caractériserions volontiers comme une non-directivité «chaude», voire «dure», et même: «dure, dure...»! C'est-à-dire qu'il a surtout été travaillé sur les conflits - par opposition donc à ce que nous appellerions une non-directivité «douce», flirtant plus ou moins avec les tentations du fusionnel. Cela nous a semblé commandé par le thème lui-même, de la confrontation (même si on laisse au mot son sens propre en français, pur de contamination franglaise) franco-allemande, et surtout par le mode de fonctionnement réel des groupes mais aussi - il faut le dire! - des équipes d'animation et de recherche (Teams) elles-mêmes... Bien plus: dans la mesure où lesdites équipes sont assez larges (de quatre à six personnes) et sans homogénéité «idéologique » (ne pouvant invoquer l'objectivité des canons épistémologiques propres à une seule discipline ou "École» théorique, ni le référent culturel d'une identité nationale commune), il s'est avéré qu'on pouvait aller parfois jusqu'à rejouer le scénario de la "guerre des chefs», ce qui peut chez certaines personnalités prendre une valeur compulsive...

Par ailleurs: quelle est la «langue de travail » adoptée? C'est dans ces termes que le lecteur non averti, qu'il soit un habitué des rencontres internationales ou non, sera tenté de poser d'abord le problème de la communication au sein du groupe. À la différence des conjonctures de bilinguisme «naturel» que la sociolinguistique prend généralement pour objet (comme en Belgique ou au Québec, par exemple), les stages francoallemands considérés sont une institution volontariste, artificielle, expérimentale. Le recrutement des participants y est individuel (et volontaire), le groupe en tant que tel a en principe une existence extrêmement limitée dans le temps, etc. Surtout, on peut considérer que les deux langues en présence (le français et l'allemand) ont l'une par rapport à l'autre un prestige socio-culturel à peu près équivalent et qu'il n'y a pas d'enjeux socio-économiques pesant massivement dans un sens ou dans l'autre sur la dynamique des groupes considérés et sur les individus qui les constituent ${ }^{5}$.

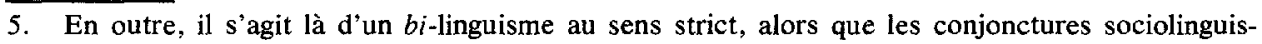
tiques "naturelles» communément appelées ainsi renvoient, dans les faits, à ce qu'il serait convenu d'appeler plutôt des "triangles glossiques" - comme cela a été souligné dans les dis- 
D'une façon générale, nous avons coutume de souligner que la recherche dont il s"agit ici s'articule selon trois axes principaux (ibid., p. 4 sqq.) :

- la dimension psychosociologique de la dynamique de groupe,

- les clivages linguistiques produits par le bilinguisme, c'est-à-dire par la présence de deux langues en contact au sein du groupe,

- la question nationale des rapports franco-allemands.

Notre titre le dit assez: c'est sur une étude de l'interférence des deux premières composantes, la composante psychosociologique et la composante (bi-)linguistique, que nous avons centré le présent «papier». Dans la mesure où c'est possible, nous négligeons ici les problèmes spécifiques, nombreux et excessivement complexes, qui sont liés à la tentative pour définir une approche ("ethnopsychologique ») de la troisième composante: le national ${ }^{6}$. Cela dit, il reste que concrètement, dans la réalité, ces trois composantes sont en interaction constante; elles ne peuvent être distinguées qu'en vertu de l'analyse et par un effort d'abstraction.

Enfin: s'agissant ici de ce qu'on pourrait appeler de la "psycho-socio-linguistique" (ibid., p. 29, 45 et passim), les premières conclusions provisoires auxquelles il est possible de parvenir ne sauraient être bien sûr des lois au sens des sciences exactes. Il ne peut guère être question non plus de diagnostic «nosographique», comme on dit en médecine, mais seulement de commencer à rassembler les éléments d'une «séméiologie», correspondant à ce que nous avons appelé après Windelband le niveau idiographique de la recherche (ibid., p. 35), qui consiste essentiellement à décrire et «isoler» des phénomènes (ou, comme on dit de plus en plus volontiers, des «effets»...). Ce sont donc les premiers points d'une liste de phénomènes et de concepts-clés, qui sont autant de «fenêtres» permettant d'éclairer la pratique des groupes bilingues, que nous abordons ici ${ }^{7}$.

$\mathrm{Si}$, par commodité, nous parlons de «dynamique des groupes bilingues», il convient de bien préciser tout de suite qu'en l'occurrence, cela ne signifie en aucun cas que les membres des groupes avec lesquels nous travaillons soient tous des "bilingues " ${ }^{8}$. La présence des unilingues n'en a absolument pas été exclue. Bien au contraire! elle est même explicitement souhaitée.

Au niveau du recrutement (input), nous avons tenu à faire savoir expressément aux futurs participants que la connaissance de l'autre langue n'était nullement une condi-

cussions que nous avons eues au sein du groupe C.L.I.C. notamment avec notre collègue P. Cadiot. - Quant aux problèmes, tout à fait différents de l'«imagologie bi-nationale», nous y avons consacré une étude sui generis: cf. J.-R. Ladmiral (1973).

6. Cf. J.-R. Ladmiral (1978), p. 6 et passim: nous y consacrerons prochainement une êtude spécifique. Par ailleurs, il est clair que le problème se poserait aussi, et d'une façon encore plus complexe, dans un contexte franco-canadien, voire franco-québécois. Au reste, ce n'est pas tout à fait sans penser à un élargissement de notre programme de recherche francoallemand dans une telle direction (l'O.F.Q.J. venant, par exemple, s'associer à l'O.F.A.J.) que nous avons tenu à publier le présent texte dans Meta, à Montréal...

7. Cf. J.-R. Ladmiral (1978), p. 38 sq. Cet inachèvement apparent d'un discours théorique essentiellement partiel et par là même pluriel, pour ainsi dire «ponctualisé » - dont la validité réside en ce qu'il est gagé sur la dialectique d'un rapport réel d'engagement vécu dans la pratique - voilà ce qui nous a amené à thématiser la notion de théorèmes : cf. J.-R. Ladmiral (1979), p. 211 sqq. et passim. À cet égard, d'un point de vue épistémologique, la pratique de groupe et celle de la traduction présentent une certaine analogie, outre leurs points de contact bien évidents ici, en l'occurrence, au plan du contenu. Sur la traduction et les phénomènes de groupe, cf. J.-R. Ladmiral (1981).

8. Il est vrai qu'il y aurait aussi dans des groupes strictement bilingues un objet d'étude possible, en quelque sorte plus "pur». Peut-être y constaterait-on que des phénomènes comme le choix de la langue ou le changement de langwe se trouveraient alors commandés par des déterminations plus psycholinguistiques que sociolinguistiques. Mais, quoi qu'il en soit, cela accentuerait encore le caractère expérimental et artificiel de la recherche et nous tenions quant à nous à ce que notre recherche, "psycho-socio-linguistique», restât plus près de la réalité de nos sociétés (cf. inf.). 
tion nécessaire pour s'inscrire aux stages organisés dans le cadre de notre programme de recherche. En procédant ainsi, nous allions à l'encontre d'un préjugé spontané. Nombreux sont en effet les individus, pourtant intéressés par ce type de travail et d'expérience, qui s'interdisent de participer à de tels stages, franco-allemands, parce qu'ils se font à eux-mêmes l'objection : «Mais! je ne sais pas l'allemand (le français)»...

Cette présence des unilingues est un point tout à fait essentiel : avant tout, parce que cela correspond à la réalité (Realität). C'est vrai des échanges franco-allemands de jeunesse: où irait-on s'ils étaient réservés dans les faits à une sorte d'aristocratie pédagogique, excessivement minoritaire et plus ou moins professionnalisée, où n'auraient leur place que ceux qui maîtrisent l'autre langue? Il y aurait là une sélection élitiste et tout à fait contraire à l'esprit du Traité d'amitié franco-allemand au terme duquel la création de l'O.F.A.J. vise à promouvoir les échanges de masse ${ }^{9}$. Mais surtout, c'est vrai plus généralement, au-delà du franco-allemand, et au-delà des échanges de jeunes en général. On conçoit mal que les rencontres internationales, qu'on voit nécessairement se multiplier de plus en plus ${ }^{10}$, ne dussent concerner que des bilingues, voire des plurilingues... En cette sorte d'affaire, il ne faut pas surestimer le facteur linguistique. Et d'ailleurs, de proche en proche, il faudrait savoir parfaitement toutes les langues!

Ce n'est donc que globalement, en tant qu'il constitue un ensemble, qu'un groupe du type de ceux dont il est question ici peut être dit bi-lingue. À vrai dire, c'est plutôt d'une dynamique de groupe di-linguistique, d'une dynamique des groupes «dilingues", que nous préférerions parler. Au sein de tels groupes, on utilise certes les deux langues; mais à côté des dits bilingues, il y a des unilingues des deux pays, "des deux langues" (des unilingues français et des unilingues allemands, à moins qu'il ne vaille mieux dire des Français unilingues et des Allemands unilingues...).

Mais, dans les faits, les frontières séparant ces deux ou trois sous-groupes différents ne sont pas aussi nettes. Des uns aux autres, il existe tout un dégradé, un continuum qui va du «bilingue» idéal (au sens d'un équilingue, qui n'existe pas) à l'unilingue absolu (qui est lui-même aussi introuvable, car on sait toujours un, deux, plusieurs mots de l'autre langue); et ce, bien sûr des deux côtés ${ }^{11}$. Entre les deux sous-groupes d'unilingues (ici francophones et germanophones), l'étanchéité interlinguistique n'est jamais totale; elle ne peut pas l'être.

D'ailleurs, il y aussi une transition insensible du verbal, en l'occurrence des bribes de l'autre langue qu'on peut appréhender, au para-verbal qui le prolonge et au non-

9. Encore convient-il de ne pas verser dans un optimisme trop naïvement internationaliste et anticonflictualiste (cf. notamment J.-R. Ladmiral, 1978, p. 43 sq. et 1973, p. 276, etc.) ni méconnaître l'importance que rêvet maintenant de plus en plus le tourisme de masse: il y a là un mode nouveau de rencontre internationale qui, à tort, n'est guère pensé comme tel par les spécialistes de la question et dont les effets sont souvent destructeurs; sur ce point, cf. notamment B. Müller \& M. Pagès (1979).

10. Au-delà du contexte qui lui a donné naissance, c'est d'ailleurs là que réside l'intérêt de notre recherche, dans son caractère transférable : du franco-allemand au plan européen (ce dont le Conseil d'Administration de l'O.F.A.J. témoigne depuis 1977 le souci explicite) mais aussi bien au-delà - comme il nous a été donné de l'entendre au symposion de Bruxelles où, à la suite de notre communication, Tibor Oláh nous a marqué son intérêt pour ce que nous avions dit en précisant qu'il lui semblait que cela s'appliquait aussi à peu de chose près au contexte des échanges soviéto-hongrois. Ainsi, toujours sur ce type de problèmes et dans la même perspective de généralisation, avons-nous été amené à faire une intervention, en collaboration avec notre collègue J.-L. Marais, dans le cadre d'un séminaire sur la communication interculturelle organisé par «SIETAR» (Society for Intercultural Education, Training and Research = Société pour l'Éducation, la Formation et la Recherche Interculturelles) au C.E.S.A. (Centre d'Études Supérieures des Affaires) à Jouy-en-Josas, le 22 février 1979, etc.

11. Ainsi avons-nous proposé de parler pragmatiquement de $\propto$ bilinguisme réel», au sens de ce fait d'expérience que connaissent bien ceux pour qui une langue seconde a cessé d'être avant tout un obstacle interlinguistique pour devenir d'abord un instrument de communication, par analogie avec l'expression de «socialisme réel » (dans notre Note sur la traduction du rire, à paraître : cf. supra note ${ }^{3}$ ). 
verbal, qui est censé le dépasser. Ainsi, par exemple, notre collègue le psychosociologue Remi Hess, avec lequel il nous est arrivé de co-animer certains stages franco-allemands ${ }^{12}$, ne connaît qu'assez imparfaitement l'allemand, mais il demande très rarement qu'on traduise pour lui ce qui se dit en allemand. Non pas seulement, ni même principalement par discrétion, ni surtout parce qu'il se désintéresserait de ce qui s'est dit. Mais parce que les quelques mots-charnières qu'il a pu «attraper» en allemand, ce qu'il sait de la vie du groupe et de son histoire au moment donné, et ce que son attention délivrée des arabesques de la parole lui fait pressentir de ce qu'il appelle lui-même les «flux libidinaux» qui circulent au sein du groupe - tout cela lui permet de "suivre ", c'est-à-dire de communiquer en s'insérant de façon adéquate dans le groupe. Autrement dit: la communication est un cas particulier du malentendu (cf. J.-R. Ladmiral, 1975, p. 16). Au-delà de certains dons de sensibilité personnels qui, sans doute, sont plus développés chez un Remi Hess que chez d'autres (ne fût-ce que pour des raisons professionnelles), il y a là quelque chose dont chacun de nous peut faire l'expérience, avec plus ou moins d'acuité, dès lors qu'on y prend un peu garde...

À lui seul, cet état de choses caractérisé par la présence des unilingues suffit à faire que les problèmes de communication «bi-lingue» qui se posent au groupe deviennent précisément des «problèmes de groupe», des problèmes $d u$ groupe, c'est-à-dire des problèmes qui mettent en jeu la dynamique de ce groupe et qu'il a à gérer en tant que groupe. C'est donc au sens plein d'une interaction substantielle entre les deux types de phénomènes mis en jeu que nous parlons ici d'une interférence de la composante linguistique du bilinguisme avec la composante psychosociologique de la dynamique de groupe.

Dans la mesure où le groupe dont nous étudions la dynamique di-linguistique n'est pas une communauté homogène d'individus bilingues, il n'est pas un mais pluriel: la différence linguistique y opère un certain nombre de clivages; et ces disparités sont un des éléments du potentiel conflictuel qui vient nourrir la dynamique du groupe - ce qui constitue une perturbation dans l'activité collective du groupe par rapport à un objectif de performance déterminé (Leistung!), mais ce qui fournit aussi au psychosociologue un dispositif permettant de mieux «travailler» avec et sur le groupe ${ }^{13}$.

Mais, comme on vient de le voir, il n'y a pas d'un côté les Allemands et de l'autre les Français, pas même les germanophones d'un côtê et les francophones de l'autre ${ }^{14}$. Si le groupe se trouve coupé en deux, c'est plus en fonction du fait qu'il y a d'une part les bilingues et de l'autre les unilingues. Encore conviendrait-il de distinguer plutôt trois sous-groupes : les bilingues, les unilingues d'une langue et les unilingues de l'autre langue (voire quatre sous-groupes, puisque les bilingues ont eux-mêmes une «langue maternelle», corrélative d'une socialisation différentielle, qui restent assez largement dominantes). Bien plus, il est apparu que, du point de vue des compétences linguistiques, le groupe tendait même à s'éparpiller finalement en presque autant de sous-groupes que d'individus, selon le degré de "bilinguisme " caractérisant chacun (au-delà du zéro et en deçà de l'infini, l'un et l'autre asymptotiques).

12. Cf. son dernier livre (R. Hess, 1981), où il fait une place assez large à notre collaboration dans ce domaine et au sein du C.R.I. (Centre de Recherches Institutionnelles).

13. On rejoint ici l'opposition bien connue entre «groupe de travail» et "groupe de base", établie par W.R. Bion. Dans le cadre d'une prochaine étude, nous reviendrons sur ce modèle proposé par le psychiatre anglais et sur ses modalités d'application au fonctionnement des groupes franco-allemands.

14. La répartition du groupe en deux sous-groupes nationaux fait même figure d'interdit et de tabou, très difficilement transgressibles. C'est un indice qui, joint à beaucoup d'autres, nous donne à penser qu'il y a là tout un complexe de problèmes inconscients encore refoulés: d'où la nécessité de ce que nous avons appelé une ethnopsychanalyse de la... "conscience nationale» (J.-R. Ladmiral, 1973, p. 265 sqq.). Quant à la répartition par groupes linguistiques, elle fait trop figure de "déplacement" (au sens psychanalytique) par rapport au tabou du national. A tous ces problèmes, encore une fois, nous consacrerons une étude sui generis. 
Il reste que, bien évidemment, on ne parle jamais «dans les-deux-langues», simultanément - de même qu'on ne prononce jamais deux segments linguistiques simultanément, conformément à l'axiome de la linéarité du langage qu'a énoncé F. de Saussure (1972, p. 103). Dans les groupes franco-allemands aussi, on parle toujours une langue ou l'autre! quand bien même on ferait alterner les deux langues fréquemment, voire régulièrement et même systématiquement. Ainsi se crée-t-il nécessairement deux réseaux de communication, deux médias uni-linguistiques, qui restent parallèles et en eux-mêmes fondamentalement imperméables l'un à l'autre.

C'est d'ailleurs cette donnée fondamentale qui définit le statut propre des bilingues dans le groupe et la façon dont ils vivent l'expérience qu'ils y font. Paraphrasant une formule sartrienne bien connue..., nous dirions volontiers qu'elle institue les bilingues comme tels; et, comme il vient d'être dit, c'est de proche en proche la place de chacun qui se trouve déterminée par rapport à cette institution linguistique. Surtout, c'est cette dualité linguistique qui met à l'ordre du jour l'échéance de la traduction, au sens élargi d'une "traduction orale " ${ }^{15}$.

La solution «technique», consistant à recourir aux compétences (c'est le cas de le dire) d'un interprète professionnel, devait être écartée dans les groupes franco-allemands du genre de ceux dont il est question ici, pour de multiples raisons - des raisons matérielles et objectives, mais aussi des raisons pédagogiques, et même politiques, etc. - que le manque de place nous empêche de développer ici ${ }^{16}$.

Dès lors, la traduction doit être gérée par le groupe lui-même, pour qui son propre fonctionnement «bilingue» devient l'un des enjeux de la dynamique psychosociologique dont il est le lieu. Du coup, la traduction devient un dispositif de groupe, un «analyseur» du groupe ${ }^{17}$. C'est-à-dire que la médiation interlinguistique (Sprachmittlung) devient l'objet et l'enjeu d'une communication de groupe. Mais soulignons bien qu'au-delà des problèmes spécifiques de ladite traduction, notre recherche prend là pour objet l'ensemble de tout ce qui concerne "l'économie linguistique» (the speech economy of a community) de cette communauté qu'est le groupe restreint, l'utilisation de plusieurs systèmes linguistiques et les changements de langues (switching et shifting) faisant partie intégrante de l'activité linguistique du groupe considéré comme un tout ${ }^{18}$.

Ces opérations de traduction - et, plus généralement, tous les problèmes liés au «bilinguisme» du groupe - les individus ont à les négocier au sein du groupe et à les re-négocier de façon permanente; la traduction est devenue un phénomène de groupe. Inversement, la différence linguistique et le dispositif de médiation interlinguistique qu'est la traduction modifient fondamentalement le fonctionnement psychosociologique

15. Il y a là un point de terminologie dont il ne sera pas traité ici, pas plus que des problèmes linguistiques de la traduction: ef. notamment J.-R. Ladmiral (1979), p. 40 sqq., etc.

16. Nous y reviendrons dans une prochaine étude, centrée sur la question de l'interprétariat dans la dynamique des groupes bi-nationaux, que nous avions annoncée précédemment (J.-R. Ladmiral, 1978, p. 3) mais qui est restée jusqu'à présent inédite dans la mesure où les perspectives de notre recherche se sont un peu modifiées, notamment sur le plan institutionnel, et dont le présent texte reprend partie en la substance.

17. C'est à dessein que nous avons fait ici plusieurs fois référence à l'Analyse institutionnelle (A.I.) : pour diverses raisons, que ce n'est guère le lieu d'....analyser en détail, ce courant de la psychosociologie française a joué un rôle tout déterminant pour la naissance et le développement de la recherche "franco-allemande" dans le cadre général de laquelle s'insère notre propre programme de recherche (ainsi que notre propre collaboration aux travaux du C.R.I.). Sur l'A.I. en général et sur le concept d'analyseur, cf. M. Authier \& R. Hess (1981).

18. Nous renvoyons à l'étude maintenant classique de D. Hymes (1962) et à toutes celles qui ont suivi sur l'ethnographie de la communication. Sur beaucoup de points nous rejoignons les recherches menées dans ce domaine, surtout aux États-Unis, et beaucoup moins en France (cf. J. Lindenfeld, 1978, p. 46 sqq.). Le manque de place nous interdit d'entamer ici la discussion des modèles proposés par D. Hymes; notons toutefois que son approche serait d'ordre plutôt «ethnosocio-linguistique» alors que, comme il a été dit plus haut, la nôtre est ici d'esprit plutôt «psychosocio-linguistique». 
du groupe (cf. J.-R. Ladmiral, 1978, p. 7); elles opèrent une re-distribution des priorités, des clivages et des forces qui sont à l'œuvre dans un groupe. Là encore, il y a interaction dialectique ou «interférence» entre ces deux composantes.

Ce facteur de perturbation supplémentaire qu'introduit le «bilinguisme» du groupe fonctionne en quelque sorte comme un accélérateur de la dynamique du groupe. Dans le champ clos du stage, il tend à exacerber les conflits (inter-personnels et collectifs), à renforcer les projections (positives et surtout négatives), à aiguiser la conscience du décalage que peut éprouver l'individu entre son comportement propre et les comportements valorisés par le groupe ${ }^{19}$, à problématiser ainsi de façon plus drastique l'insertion de chacun dans le groupe, etc. Il se produit notamment une inflation du «non-dit». Ou pour le dire autrement, en usant d'une autre métaphore, c'est un miroir déformant, qui fournit une caricature des réalités habituelles et familières de la dynamique de groupe; mais, comme on dit, il arrive souvent que la caricature soit «plus vraie que nature», plus profondément vraie. De même ce miroir di-linguistique jette une lumière nouvelle et très éclairante sur les phénomènes de groupe ${ }^{20}$.

De la même façon, le vécu d'un stage de dynamique de groupe est parfaitement artificiel, tout à fait aberrant par rapport au vécu normal ou «naturel» des participants en dehors du stage. Mais cette image déformée, cette «simulation» caricaturale fait d'autant mieux ressortir certains éléments de la réalité qui n'avaient pas été jusqu'alors pris en compte - qu'ils soient restés inaperçus parce que trop éparpillés dans la diversité étourdissante et foisonnante de la vie quotidienne et/ou qu'ils aient été plus ou moins consciemment scotomisés ou refoulés jusqu'à ce que, dans la situation de crise artificiellement entretenue et sans enjeux réels que constitue le stage, ils puissent enfin trouver à s'exprimer (à tout le moins plus clairement).

Concrètement, le fait que la traduction soit devenue l'affaire du groupe, voilà qui pose différents problèmes. La solution technique de l'interprète étant écartée, cela veut dire qu'il n'y a pas dans le groupe une personne (ou deux) investie(s) institutionnellement de la fonction (de la "charge», aux divers sens que prend le mot) d'y assurer la médiation interlinguistique. Cela contribue sans doute à démystifier en partie ce que nous avons appelé l'illusion ou le fantasme de «la transparence traductive» (J.-R. Ladmiral, 1979 , p. 230 sqq.). Ainsi réintégrée dans le contexte d'une pratique sociale («psychosociale"), la traduction ne peut plus être réduite à l'application subalterne d'introuvables «techniques», comme le voudrait une erreur assez répandue (ibid., p. 18 et passim). Mais dès lors, qui va traduire?

Les «bilingues», bien sûr. Mais lesquels? dans quelle proportion et dans quelles conditions? comment? et pourquoi?... L'expérience montre que les bilingues $n$ 'assurent pas tous également cette fonction de traduction, ni aux mêmes moments. Au fur et à mesure que se déroule le temps du stage, certains traduisent de plus en plus, d'autres de moins en moins (de même que certains parlent de plus en plus l'autre langue, d'autres de moins en moins, etc.). Certains se refusent à traduire en général, à traduire les autres, à se traduire eux-mêmes. D'autres au contraire lancent, pourrait-on dire, une véritable «O.P.A.» sur la fonction de traducteur: ils ne cessent de parler la parole des autres dans une langue différente, etc. Quelles sont les motivations des uns et des autres? quelle est la part des contraintes «objectives», au niveau des mécanismes psycholinguistiques de leur(s) fonctionnement(s) bilingue(s), dans leurs comportements respectifs?... et qu'est-ce que cela «induit» dans le groupe?

19. Le groupe bi-national met en concurrence plusieurs modèles culturels d'identification et libère ainsi de fortes charges d'ambivalence, laquelle peut se traduire par des comportements de conflits, de séduction, etc. Mais, là encore, on rejoint le problème du national...

20. C'est ainsi qu'il a pu fonctionner comme dispositif d'("auto-») formation collective et accélérée, "sur le tas", pour les chercheurs-animateurs qui, au sein de ces programmes, n'étaient pas psychosociologues au départ. Plus généralement, sur l'importance de la pluridisciplinarité en cette affaire, cf. J.-R. Ladmiral (1978), p. 16 sqq. 
À la question des motivations, il n'est guère possible pour l'instant, dans la phase actuelle de notre recherche, de répondre de manière simple et univoque. On est renvoyé à l'histoire individuelle de chacun et à la façon dont il vit son rapport au groupe. Il y a aussi une histoire "individuelle» de chaque groupe, de chaque stage : car les uns et les autres ne sont pas interchangeables. Au-delà d'une certaine sensibilisation, d'une réelle expérience et d'une relative clairvoyance que l'on peut acquérir dans la «pratique de groupe ", il n'existe pas là plus qu'en matière de traduction des «techniques » linéairement applicables, ni des mécanismes automatiques (cf. J.-R. Ladmiral, 1978, p. 21 sq.). C'est pourquoi nous avons parlé d'une approche «idiographique» (cf. sup.) ou encore clinique (J.-R. Ladmiral, 1978, p. 34), dont, encore une fois, le manque de place nous empêche ici d'exposer le programme au-delà de ces quelques indications initiales.

Les motivations à traduire peuvent être très diverses. Certains achètent ainsi leur droit de parole, soit qu'ils soient «inhibés» (timides), soit qu'au contraire ils tendent à occuper un temps de parole supérieur: ils traduisent et, à cette occasion, ils glissent le codicille d'une petite remarque personnelle ou l'intervention massive d'un commentaire critique, voire d'une prise de position manipulatoire. Certains se font excuser leur présence, pour ainsi dire, en traduisant les autres. Certains, parmi les étudiants germanistes français (ou romanistes allemands) par exemple, voient là une bonne occasion de s'entraîner et s'imposent cet exercice pour améliorer leurs performances dans l'autre langue ${ }^{21}$. Pour certains, la traduction devient non seulement un moyen d'échange, mais aussi un moyen de pression, de chantage; elle peut servir à prendre le pouvoir dans le groupe, etc. C'est, par exemple, l'un des aspects que peut revêtir la dialectique complexe qui se joue entre pouvoir et communication dans les groupes bilingues. D'une façon générale, il y a là, parallèlement à la question des motivations, toute une problématique du pouvoir linguistique, qui va bien au-delà de la seule question des motivations de l'individu à traduire pour le groupe...22

Mais il manquerait un point essentiel à ce rapide survol si nous omettions de souligner qu'à renoncer à la solution dite technique de l'interprète, comme nous le faisons dans les stages mis en place dans le cadre de notre programme de recherche, on s'affranchit du même coup aussi du formalisme d'une traduction systématique de toutes les interventions, avec toutes les pesanteurs que cela entraîne.

Va-t-on tout traduire? y compris les invectives et autres interruptions ( $Z$ wischenrufe) ?... A chaque fois, la traduction introduit un retard, comme un temps mort; elle ajourne le dialogue direct. Elle a un effet de freinage qui bride toute spontanéité et, sans qu'on s'en aperçoive d'abord, elle menace finalement de bloquer toute discussion réelle. Au bout du compte, le dispositif de la traduction risque de peser tellement sur la dynamique du groupe qu'elle rendrait impossible la communication qu'elle a pour fonction de promouvoir. Il suffit de penser au retard que, de façon analogue et parallèle, les grandes Organisations internationales ne cessent d'accumuler par rapport à leur propre actualité politique donnant naissance aux textes qu'elles entreprennent elles-mêmes de faire traduire...

Et si on ne traduit pas tout, on fait nécessairement une sélection. Mais au nom de quels critères? et qui la fera? et puis cela ne revient-il pas précisément à exclure les unilingues de la communication, à les «minoriser»? Bien plus, dans les faits, c'est l'un

21. C'est particulièrement net dans certains stages, comme celui que nous avons co-animé avec Remi Hess et Ralf Dantscher à Berlin en septembre 1976 et dont nous avons publié une brève analyse (R. Hess \& J.-R. Ladmiral, 1977), sans toutefois thématiser cet aspect du problème autrement que par une indication allusive au passage (p. 43).

22. Conjointement aux autres problèmes évoqués plus haut, cette problématique sera traitée dans le cadre de notre prochain ouvrage sur la dynamique des groupes bilingues, précédemment annoncé, où nous ferons un bilan concernant le programme de recherche franco-allemand dont nous avons la responsabilité, au-delà des «premières conclusions provisoires" qui, dans le présent «papier», ne font encore que baliser le champ d'étude que ladite recherche nous a permis de définir. 
des deux sous-groupes des unilingues qui va se trouver exclu, car il y aura bien sûr une langue dominante, sinon pour tout le stage du moins à certains moments, dans certains sous-groupes, selon certains sujets, certains lieux, etc.

On retrouve là, semble-t-il, la constellation sociolinguistique bien connue qui articule le bilinguisme en langue dominante et langue dominée; mais avec cette différence fondamentale que, dans un groupe comme ceux avec lesquels nous travaillons, la domination d'une langue ou l'autre est sans cesse re-négociable - en fonction, il est vrai, de bien des transversalités et autres paramètres conditionnant l'ensemble. Mais ces divers paramètres sont assez nombreux pour que la marge de manœuvre (c'est-à-dire aussi de «manœuvres », au pluriel, et de manipulation...) dont disposent les individus reste assez large. C'est là encore un aspect, complémentaire du précédent, du jeu dialectique où interfèrent pouvoir et communication dans la dynamique des groupes bilingues. Le renversement (ou la stabilisation) d'un tel rapport de domination passe le plus souvent par des relais et des opérations de traduction. À cette occasion, mais aussi au-delà et d'une façon beaucoup plus générale, on mesure à quel point la traduction est un enjeu et un mode d'intervention psychosociologique (cf. J.-R. Ladmiral, 1980) massif sur la dynamique des groupes bilingues.

\section{BIBLIOGRAPHIE}

AUTHIER, Michel et Remi HESS (1981): L'Analyse institutionnelle, Paris, P.U.F., «Que Sais-je?» $\mathrm{n}^{\circ} 1968$.

FISHMAN, Joshua A. (ed.) (1970): Readings in the Sociology of Language, La HayeParis, Mouton.

GUIGOU, Jacques (1975) : «La stagification», Éducation permanente, n 31, Novembre-Décembre, p. 5-25.

HESS, Remi (1981) : Le Temps des médiateurs, Paris, Anthropos.

HESS, Remi et Jean-René LADMIRAL (1977) : "Émancipation personnelle et sociale", Allemagnes d'aujourd'hui, $\mathrm{n}^{\circ}$ 60, Novembre-Décembre, p. 43-47.

HYMES, Dell H. (1962): "The Ethnography of Speaking», in T. Gladwin \& W.C. Sturtevant (eds.), Anthropology and Human Behavior, Washington D.C., Anthropological Society, p. 13-53. Repris in J.A. Fishman (1970), p. 99-138.

LADMIRAL, Jean-René (1973): "Culture française et stéréotypes à l'égard de l'Allemagne - De l'ethnopsychologie à la double culture", Revue d'Allemagne, t. $\mathrm{V} / \mathrm{n}^{\circ} 2$, avril-juin, p. 238-278.

- (1975): «Linguistique et pédagogie des langues étrangères», in Langages, $\mathrm{n}^{\circ} 39$, septembre, p. 5-18.

- (1978): "Pour une dynamique des groupes bi-nationaux - Préliminaires méthodologiques », Langage et Société, $\mathrm{n}^{\circ}$ 3, février, p. 3-47.

(1979): Traduire : théorèmes pour la traduction, Paris, Payot.

(1980): "Philosophie de la traduction et linguistique d'intervention», Traduzione tradizione, numéro spécial de la revue Lectures, $n^{\circ} 4-5$, Agosto, p. 11-41.

LADMIRAL, Jean-René et Henri MESCHONNIC (1981): (sous la dir. de) numéro spécial sur La traduction de la revue Langue française, $\mathrm{n}^{\circ} 51$, septembre.

DE LAUNAY, Marc B. (1981) : "Le traducteur médusé», in J.-R. Ladmiral et H. Meschonnic, p. 53-62.

LINDENFELD, Jacqueline (1978): «L'ethnographie de la communication a-t-elle un sens pour les linguistes?», Langage et Société, $\mathrm{n}^{\circ} 5$, septembre, p. 45-52.

MARC-LIPIANSKY, Edmond (1978): "Groupe et identité ", in Identités collectives et relations inter-culturelles, sous la dir. de G. Michaud, Bruxelles, Complexe, p. 59-88.

MƯLLER, Burkhard et Max PAGÈS (1979): «Existentielle Animation - Gedanken zur einer Neuorientierung der Begegnung in Gruppen", Deutsche Jugend, Jhg. XXVII / Hft. 7, Juli, SS. 301-310. Reprise plus détaillée chez Suhrkamp, dans la série Friedensanalysen (édition Suhrkamp, Bd. 784).

NELDE, Peter Hans (ed.) (1980) : Sprachkontakt und Sprachkonflikt, Wiesbaden, Steiner, (Zeitschrift für Dialektologie und Linguistik: Beihefte, hrsg. v. J. Göschel u. W. H. Veith, Heft 32). 
DE SAUSSURE, Ferdinand (1972): Cours de linguistique générale, éd. T. de Mauro, Paris, Payot, «Payothèque», rééd.

SCHERFER, Peter (1977) : «Lernhemmungen und ihre Überwindung in Sprachlehrprozessen auf Gegenseitigkeit in deutsch-französischen Gruppen», Zielsprache Französisch, Heft 2, SS. 53-65. 